\title{
Dynamical Systems Method (DSM) for general nonlinear equations
}

\author{
A.G. Ramm \\ Mathematics Department, Kansas State University, Manhattan, KS 66506-2602, USA
}

Received 18 July 2007; accepted 23 July 2007

\begin{abstract}
If $F: H \rightarrow H$ is a map in a Hilbert space $H, F \in C_{\mathrm{loc}}^{2}$, and there exists $y$ such that $F(y)=0, F^{\prime}(y) \neq 0$, then equation $F(u)=0$ can be solved by a DSM (dynamical systems method). This method yields also a convergent iterative method for finding $y$, and this method converges at the rate of a geometric series. It is not assumed that $y$ is the only solution to $F(u)=0$. A stable approximation to a solution of the equation $F(u)=f$ is constructed by aSM when $f$ is unknown but $f_{\delta}$ is known, where $\left\|f_{\delta}-f\right\| \leq \delta$.

(C) 2007 Elsevier Ltd. All rights reserved.
\end{abstract}

MSC: $47 \mathrm{H} 15 ; 47 \mathrm{H} 20 ; 65 \mathrm{H} 10 ; 65 \mathrm{~J} 15 ; 65 \mathrm{~N} 20$

Keywords: Nonlinear operator equations; Iterative methods; DSM—dynamical systems method

\section{Introduction}

In this paper a method for solving a fairly general class of nonlinear operator equations $F(u)=0$ in a Hilbert space is proposed, its convergence is proved, and an iterative method for solving the above equation is constructed. Convergence of the iterative method is also proved. These results are based on the following assumptions: (a) the above equation has a solution $y$, possibly non-unique, (b) $F \in C_{\mathrm{loc}}^{2}$, and (c) $F^{\prime}(y) \neq 0$. No restrictions on the rate of growth of the nonlinearity are imposed. The literature on methods for solving nonlinear equations is large (see, e.g., [1] and references therein). Most of the results obtained so far are based on Newton-type methods and their modifications. There is also a well-developed theory for equations with monotone operators $[4,3]$. The method used in this paper is a version of the dynamical systems method which is studied in $[2,3]$. The idea of this method is described briefly below.

Let $F: H \rightarrow H$ be a map in a Hilbert space. Assume that equation

$$
F(u)=0
$$

has a solution $y$, possibly non-unique, and

$$
F^{\prime}(y) \neq 0,
$$

E-mail address: ramm@math.ksu.edu. 
where $F^{\prime}$ is the Fréchet derivative of $F$. This assumption means that $F^{\prime}(y)$ is not equal to zero identically on $H$. Thus, it is a weak assumption. Assume that $F \in C_{\text {loc }}^{2}$, i.e.,

$$
\sup _{u \in B\left(u_{0}, R\right)}\left\|F^{(j)}(u)\right\| \leq M_{j}(R) \quad 0 \leq j \leq 2,
$$

where $u_{0} \in H$ is a given element, $R>0$, and no restrictions on the growth of $M_{j}(R)$ as $R$ grows are imposed. This means that the nonlinearity $F$ can grow arbitrarily fast as $\left\|u-u_{0}\right\|$ grows. It is known that under these assumptions Eq. (1) may have no solutions. Thus, we have assumed that a solution $y$ to (1) exists.

We do not assume that $F^{\prime}(u)$ has a bounded inverse operator. Therefore the standard Newton-type methods are not applicable. The dynamical system method (DSM) consists of finding an operator $\Phi$ such that the problem

$$
\dot{u}=\Phi(t, u), \quad u(0)=u_{0} \quad t \geq 0, \quad \dot{u}=\frac{\mathrm{d} u}{\mathrm{~d} t},
$$

has a unique global solution $u(t)$, there exists $u(\infty)$, and $F(u(\infty))=0$. To ensure the unique local solvability of (4) we assume that

$$
\|\Phi(t, u)-\Phi(t, v)\| \leq L(R)\|u-v\| \quad \forall u, v \in B\left(u_{0}, R\right) .
$$

Then the global existence of the unique local solution holds if $\sup _{t \geq 0}\|u(t)\|<\infty$.

The results of this paper can be summarized in two theorems. Let us define

$$
A:=F^{\prime}(u(t)), \quad T:=A^{*} A, \quad T_{a}:=T+a I ; \quad \tilde{A}:=F^{\prime}(y), \quad \widetilde{T}=\widetilde{A}^{*} \widetilde{A} .
$$

Assume that $a(t)$ is a positive monotonically decaying function,

$$
a(t)>0, \quad \lim _{t \rightarrow \infty} a(t)=0, \quad \frac{|\dot{a}|}{a} \leq \frac{1}{2} .
$$

Theorem 1. If a solution y to Eq. (1) exists, possibly is non-unique, and assumptions (2) and (3) hold, then $y=u(\infty)$, where $u(t)$ solves the following DSM problem:

$$
\dot{u}=-T_{a(t)}^{-1}\left[A^{*} F(u(t))+a(t)(u(t)-z)\right], \quad u(0)=u_{0},
$$

and where $z$ and $u_{0}$ are suitably chosen.

Theorem 2. Under the assumptions of Theorem 1, the iterative process

$$
u_{n+1}=u_{n}-h_{n} T_{a_{n}}^{-1}\left[A^{*}\left(u_{n}\right) F\left(u_{n}\right)+a_{n}\left(u_{n}-z\right)\right], \quad u_{0}=u_{0},
$$

where $h_{n}>0$ and $a_{n}>0$ are suitably chosen, generates the sequence $u_{n}$ converging to $y$.

Remark 1. The suitable choices of $z$ and $u_{0}$ are discussed in the proof of Theorem 1. The suitable choices of $a_{n}$ and $h_{n}$ are discussed in the proof of Theorem 2.

Remark 2. Essentially, Theorem 1 says that any solvable operator equation with $C_{\text {loc }}^{2}$ operator, satisfying only a weak assumption (2), can be solved by a DSM. Condition (2) means that the range of the linear operator $F^{\prime}(y)$ contains at least one non-zero element. This allows $F^{\prime}(y)$ to have infinite-dimensional null-space.

Although we do not give an algorithmic choice of $z$ without making an additional source-type assumption, we can claim that the set of suitable $z$, i.e., the $z$ for which the conclusion of Theorem 1 holds, is dense in $H$ under some verifiable assumptions. For example, this is the case if $F^{\prime}(y)$ is compact and its range is dense in $H$.

Under some additional assumptions one can give an algorithmic choice of $z$. For example, one can choose $z=0$ if $y=\tilde{T} v$, where $\tilde{T}$ is defined in (5) and $v$ is sufficiently small. This is verified by an argument similar to the one used in the proof of Theorem 1 in Section 2. Such an assumption was referred to above as a source-type assumption.

In Section 2 we prove Theorems 1 and 2. In Sections 3 and 4 we study the stability of the solution. In the proofs we use the following lemmas.

Lemma 1. Assume that $g(t) \geq 0$ is a $C^{1}([0, \infty))$ function satisfying the inequality 


$$
\dot{g} \leq-\gamma(t) g+\alpha(t) g^{2}+\beta(t), \quad t \geq 0, \quad \dot{g}:=\frac{\mathrm{d} g}{\mathrm{~d} t},
$$

where $\gamma, \alpha$ and $\beta$ are nonnegative continuous functions. Assume that there exists $\mu \in C^{1}([0, \infty)), \mu>0$, $\lim _{t \rightarrow \infty} \mu(t)=\infty$, such that

$$
\text { (i) } \alpha(t) \leq \frac{\mu(t)}{2}\left(\gamma(t)-\frac{\dot{\mu}(t)}{\mu(t)}\right), \quad \text { (ii) } \beta \leq \frac{1}{2 \mu(t)}\left(\gamma(t)-\frac{\dot{\mu}(t)}{\mu(t)}\right), \quad \text { (iii) } g(0) \mu(0)<1 .
$$

Then any solution to $(9)$ exists on $[0, \infty)$ and

$$
0 \leq g(t)<\frac{1}{\mu(t)}, \quad t \in[0, \infty)
$$

Lemma 1 is proved in [2, pp. 66-70].

Lemma 2. Let $g_{n+1} \leq \gamma g_{n}+p g_{n}^{2}, g_{0}:=m>0,0<\gamma<1$, $p>0$. If $m<\frac{q-\gamma}{p}$, where $\gamma<q<1$, then $\lim _{n \rightarrow \infty} g_{n}=0$, and $g_{n} \leq g_{0} q^{n}$.

Proof of Lemma 2. Estimate $g_{1} \leq \gamma m+p m^{2} \leq q m$ holds if $m \leq \frac{q-\gamma}{p}, \gamma<q<1$. Assume that $g_{n} \leq g_{0} q^{n}$. Then

$$
g_{n+1} \leq \gamma g_{0} q^{n}+p\left(g_{0} q^{n}\right)^{2}=g_{0} q^{n}\left(\gamma+p g_{0} q^{n}\right)<g_{0} q^{n+1},
$$

because $\gamma+p g_{0} q^{n}<\gamma+p g_{0} q \leq q$. Lemma 2 is proved.

\section{Proofs}

Proof of Theorem 1. If $F^{\prime}(y):=A$ is linear and $A \neq 0$, then there exists $v_{1} \neq 0, v_{1}=\widetilde{T} v$. By the linearity of $\widetilde{T}$, every element $c v_{1}$ belongs to the range of $\widetilde{T}$ for any constant $c$, because $c v_{1}=\widetilde{T}(c v)$. Therefore there exists a $z$ such that

$$
y-z=\widetilde{T} v,
$$

where $\|v\|>0$ can be chosen arbitrarily small. How small $\|v\|$ should be chosen will become clear later. Let

$$
u(t)-y:=w(t), \quad\|w(t)\|:=g(t) .
$$

Write Eq. (7) as

$$
\dot{w}=-T_{a(t)}^{-1}\left[A^{*}(F(u)-F(y))+a(t) w+a(t)(y-z)\right],
$$

and use the formula

$$
F(u)-F(y)=A w+K,
$$

where

$$
\|K\| \leq \frac{M_{2} g^{2}}{2} .
$$

Then

$$
\dot{w}=-w-T_{a(t)}^{-1} A^{*} K-a(t) T_{a(t)}^{-1} \widetilde{T} v .
$$

Multiply this equation by $w$ in $H$ and use the estimate $\left\|T_{a}^{-1} A^{*}\right\| \leq \frac{1}{2 \sqrt{a}}, a>0$, (see [2]), to get

$$
g \dot{g} \leq-g^{2}+\frac{1}{2 \sqrt{a(t)}} \frac{M_{2} g^{3}}{2}+a(t)\left\|\left(T_{a(t)}^{-1}-\widetilde{T}_{a(t)}^{-1}+\widetilde{T}_{a(t)}^{-1}\right) \widetilde{T}\right\|\|v\| g .
$$

If $a>0$ then $\left\|\widetilde{T}_{a}^{-1} \widetilde{T}\right\| \leq 1$,

$$
a\left\|T_{a}^{-1}\right\| \leq 1, \quad a\left\|\left(T_{a}^{-1}-\widetilde{T}_{a}^{-1}\right) \widetilde{T}\right\|=a\left\|T_{a}^{-1}\left(A^{*} A-\widetilde{A}^{*} \widetilde{A}\right) \widetilde{T}_{a}^{-1} \widetilde{T}\right\| \leq 2 M_{1} M_{2} g .
$$


Collecting the above estimates and choosing $\|v\|$ so that

$$
2 M_{1} M_{2}\|v\| \leq \frac{1}{2},
$$

we obtain

$$
\dot{g} \leq-\frac{g}{2}+\frac{c_{0} g^{2}}{\sqrt{a(t)}}+a(t)\|v\|, \quad c_{0}:=\frac{M_{2}}{4} .
$$

Apply Lemma 1 to (14). Here $\gamma=\frac{1}{2}, \alpha=\frac{c_{0}}{\sqrt{a(t)}}, \beta=a(t)\|v\|$. Let

$$
\mu(t)=\frac{\lambda}{\sqrt{a(t)}}, \quad \lambda=\text { const }>0 .
$$

Condition (i) of Lemma 1 holds if $\frac{c_{0}}{\sqrt{a(t)}} \leq \frac{\lambda}{2 \sqrt{a(t)}}\left(\frac{1}{2}-\frac{1}{2} \frac{|\dot{a}|}{a}\right)$. This inequality holds if $\lambda \geq 8 c_{0}$; see the last assumption (6).

Condition (iii) holds if $g(0) \frac{\lambda}{\sqrt{a(0)}}<1$. This inequality holds (for any initial value $g(0)=\left\|u_{0}-y\right\|$ ) if $a(0)$ is sufficiently large.

Condition (ii) holds if $\sqrt{a(t)}\|v\| \leq \frac{1}{8 \lambda}$, where we have used the last assumption (6) again. This inequality holds if

$$
8 \lambda \sqrt{a(0)}\|v\| \leq 1 . \quad(*)
$$

Recall that $a(0) \geq a(t)$ due to the monotonicity of $a(t)$. Inequality $(*)$ holds if $\|v\|$ is sufficiently small. Thus, if $\|v\|$ is sufficiently small, then, by Lemma 1 , we get $g(t)<\frac{\sqrt{a(t)}}{\lambda}$, so $\|u(t)-y\| \leq \frac{\sqrt{a(t)}}{\lambda} \rightarrow 0$ as $t \rightarrow \infty$.

Theorem 1 is proved.

Proof of Theorem 2. Let $w_{n}:=u_{n}-y, g_{n}:=\left\|w_{n}\right\|$. As in the proof of Theorem 1, we assume $2 M_{1} M_{2}\|v\| \leq \frac{1}{2}$ and rewrite (8) as

$$
w_{n+1}=w_{n}-h_{n} T_{a_{n}}^{-1}\left[A^{*}\left(u_{n}\right)\left(F\left(u_{n}\right)-F(y)\right)+a_{n} w_{n}+a_{n}(y-z)\right], \quad w_{0}=\left\|u_{0}-y\right\| .
$$

Using the Taylor formula

$$
F\left(u_{n}\right)-F(y)=A\left(u_{n}\right) w_{n}+K\left(w_{n}\right), \quad\|K\| \leq \frac{M_{2} g_{n}^{2}}{2},
$$

the estimate

$$
\left\|T_{a_{n}}^{-1} A^{*}\left(u_{n}\right)\right\| \leq \frac{1}{2 \sqrt{a_{n}}},
$$

and the formula

$$
y-z=\tilde{T} v,
$$

we get

$$
w_{n+1}=\left(1-h_{n}\right) w_{n}-h_{n} T_{a_{n}}^{-1} A^{*}\left(u_{n}\right) K\left(w_{n}\right)-h_{n} a_{n} T_{a_{n}}^{-1} \widetilde{T} v .
$$

Taking into account that $\left\|\widetilde{T}_{a}^{-1} \widetilde{T}\right\| \leq 1$, and $a\left\|T_{a}^{-1}\right\| \leq 1$ if $a>0$, we obtain

$$
\left\|T_{a_{n}}^{-1} \tilde{T} v\right\| \leq\left\|\left(T_{a_{n}}^{-1}-\widetilde{T}_{a_{n}}^{-1}\right) \widetilde{T}\right\|\|v\|+\|v\|,
$$

and

$$
\left\|\left(T_{a_{n}}^{-1}-\widetilde{T}_{a_{n}}^{-1}\right) \widetilde{T}\right\|=\left\|T_{a_{n}}^{-1}\left(\widetilde{T}_{a_{n}}-T_{a_{n}}\right) \widetilde{T}_{a_{n}}^{-1} \widetilde{T}\right\| \leq \frac{2 M_{1} M_{2} g_{n}}{a_{n}}:=\frac{c_{1} g_{n}}{a_{n}} .
$$

Let $c_{0}:=\frac{M_{2}}{4}$. Then we obtain from (15) the following inequality:

$$
g_{n+1} \leq\left(1-h_{n}\right) g_{n}+\frac{c_{0} h_{n} g_{n}^{2}}{\sqrt{a_{n}}}+c_{1} h_{n}\|v\| g_{n}+h_{n} a_{n}\|v\| .
$$


We have assumed in the proof of Theorem 1 that

$$
c_{1}\|v\| \leq \frac{1}{2} \text {. }
$$

Thus

$$
g_{n+1} \leq\left(1-\frac{h_{n}}{2}\right) g_{n}+\frac{c_{0} h_{n}}{\sqrt{a_{n}}} g_{n}^{2}+h_{n} a_{n}\|v\| .
$$

Choose $a_{n}=16 c_{0}^{2} g_{n}^{2}$. Then $\frac{c_{0} g_{n}}{\sqrt{a_{n}}}=\frac{1}{4}$, and

$$
g_{n+1} \leq\left(1-\frac{h_{n}}{4}\right) g_{n}+16 c_{0}^{2} h_{n}\|v\| g_{n}^{2}, \quad g_{0}=\left\|u_{0}-y\right\| \leq R,
$$

where $R>0$ is defined in (3). Take $h_{n}=h \in(0,1)$ and choose $g_{0}:=m$, where

$$
m<\frac{q+h-1}{16 c_{0} h\|v\|} .
$$

Here $q \in(0,1)$ and $q+h>1$. Then Lemma 2 implies

$$
\left\|u_{n}-y\right\| \leq g_{0} q^{n} \rightarrow 0 \text { as } n \rightarrow \infty \text {. }
$$

Theorem 2 is proved.

\section{Stability of the solution}

Assume that $F(y)=f$, where the exact data $f$ are not known but the noisy data $f_{\delta}$ are given, $\left\|f_{\delta}-f\right\| \leq \delta$. Then the DSM yields a stable approximation of the solution $y$ if the stopping time $t_{\delta}$ is properly chosen. The DSM is similar to (7):

$$
\dot{u}_{\delta}=-T_{a(t)}^{-1}\left[A^{*}\left(F\left(u_{\delta}(t)\right)-f_{\delta}\right)+a(t)\left(u_{\delta}(t)-z\right)\right], \quad u_{\delta}(0)=u_{0},
$$

Let

$$
w_{\delta}:=u_{\delta}(t)-y, \quad g_{\delta}(t):=\left\|w_{\delta}\right\| .
$$

As in the proof of Theorem 1 we derive an inequality similar to (14):

$$
\dot{g}_{\delta} \leq-\frac{g_{\delta}}{2}+\frac{c_{0} g_{\delta}^{2}}{\sqrt{a(t)}}+a(t)\|v\|+\frac{\delta}{2 \sqrt{a(t)}}, \quad c_{0}:=\frac{M_{2}}{4},
$$

and apply Lemma 1 . The only difference is in checking condition (ii) of Lemma 1 . This condition now takes the form

$$
a(t)\|v\|+\frac{\delta}{2 \sqrt{a(t)}} \leq \frac{\sqrt{a(t)}}{8 \lambda} .
$$

This condition can be satisfied only for $t \in\left[0, t_{\delta}\right]$, where $t_{\delta}<\infty$. The stopping time $t_{\delta}$ can be determined, for example, from the equation

$$
4 \lambda \frac{\delta}{a(t)}=\frac{1}{2}
$$

provided that $v$ is chosen sufficiently small, so that

$$
8 \lambda \sqrt{a(0)}\|v\| \leq \frac{1}{2} \quad \text { and } \quad \lambda \geq 8 c_{0},
$$

as in the proof of Theorem 1 . If $t_{\delta}$ is so determined, then, by Lemma 1, we have

$$
g_{\delta}\left(t_{\delta}\right)<\frac{\sqrt{a\left(t_{\delta}\right)}}{\lambda} \rightarrow 0 \text { as } \delta \rightarrow 0 .
$$

Let us formulate the result. 


\section{ARTICLE IN PRESS}

Theorem 3. Let $u_{\delta}:=u_{\delta}\left(t_{\delta}\right)$, where $u_{\delta}(t)$ solves problem (17) and $t_{\delta}$ is chosen as above. Then $\lim _{\delta \rightarrow 0}\left\|u_{\delta}-y\right\|=0$.

\section{Stability of the iterative solution}

Assume that the equation is $F(u)=f, f$ is unknown, but the "noisy datum" $f_{\delta}$ is known, such that $\left\|f_{\delta}-f\right\| \leq \delta$. Consider an iterative process similar to $(8)$ :

$$
v_{n+1}=v_{n}-h_{n} T_{a_{n}}^{-1}\left[A^{*}\left(v_{n}\right)\left(F\left(v_{n}\right)-f_{\delta}\right)+a_{n}\left(v_{n}-z\right)\right], \quad v_{0}=u_{0} .
$$

Let

$$
w_{n}:=v_{n}-y, \quad\left\|w_{n}\right\|:=\psi_{n},
$$

and choose $h_{n}=h$ independent of $n, h \in(0,1)$. Later we impose a positive lower bound on $h$; see formula (24) below. An inequality similar to (16), see also (18), is

$$
\psi_{n+1} \leq \gamma \psi_{n}+p \psi_{n}^{2}+\frac{h \delta}{2 \sqrt{a_{n}}}, \quad \psi_{0}=\left\|u_{0}-y\right\| .
$$

Here

$$
\gamma:=1-\frac{h}{4}, \quad p:=16 c_{0}^{2}\|v\|, \quad a_{n}=16 c_{0}^{2} \psi_{n}^{2} .
$$

We stop iterations in (20) when $n=n(\delta)$, where $n(\delta)$ is the largest integer for which the following inequality:

$$
\frac{h \delta}{2 \sqrt{a_{n}}} \leq \kappa \gamma \psi_{n}, \quad \kappa \in\left(0, \frac{1}{3}\right)
$$

holds. One can use formula (21) for $a_{n}$ and rewrite this inequality as follows:

$$
\frac{h \delta}{8 c_{0} \kappa \gamma} \leq \psi_{n}^{2}, \quad \kappa \in\left(0, \frac{1}{3}\right) .
$$

If (22) holds, then (20) implies

$$
\psi_{n+1} \leq(1+\kappa) \gamma \psi_{n}+p \psi_{n}^{2}, \quad(1+\kappa) \gamma<1,
$$

and the conditions

$$
\gamma=1-\frac{h}{4}, \quad 0<\kappa<\frac{1}{3}, \quad h \in\left(\frac{4 \kappa}{1+\kappa}, 1\right)
$$

imply that

$$
(1+\kappa) \gamma<1 .
$$

If

$$
\psi_{0}<\frac{q-(1+\kappa) \gamma}{p}, \quad \text { where }(1+\kappa) \gamma<q<1, \quad \gamma=1-\frac{h}{4},
$$

then (23) and Lemma 2 imply

$$
\psi_{n} \leq \psi_{0} q^{n}
$$

provided that

$$
n<n(\delta), \quad(1+\kappa) \gamma<q<1, \quad 0<\kappa<\frac{1}{3},
$$

where $n(\delta)$ is the largest integer for which inequality (22) holds. We have

$$
\lim _{\delta \rightarrow 0} n(\delta)=\infty
$$


Thus

$$
\lim _{\delta \rightarrow 0} \psi_{n(\delta)}=0 .
$$

We have proved the following result.

Theorem 4. Let the assumptions of Theorem 1 and conditions (22), (24) and (25) hold, and $\psi_{n}$ be defined by (19). Then relations (26) and (28) hold, so

$$
\lim _{\delta \rightarrow 0}\left\|v_{n(\delta)}-y\right\|=0
$$

\section{References}

[1] J. Ortega, W. Rheinboldt, Iterative Solution of Nonlinear Equations in Several Variables, SIAM, Philadelphia, 2000.

[2] A.G. Ramm, Inverse Problems, Springer, New York, 2005.

[3] A.G. Ramm, Dynamical Systems Method for Solving Operator Equations, Elsevier, Amsterdam, 2007.

[4] E. Zeidler, Nonlinear Functional Analysis and its Applications, II-B, Springer, New York, 1985. 